\title{
High Quality Imaging for Space VLBI Observations with Ultra High Angular Resolution
}

\author{
Sergey Likhachev \\ Astro Space Center, Lebedev Physical Institute, Moscow, Russia
}

\begin{abstract}
This presentation focuses on the problems associated with obtaining high quality images from a high orbiting space VLBI (SVLBI) mission. SVLBI intensifies and magnifies all imaging problems, making these problems clearer to understand, though much harder to solve.
\end{abstract}

\section{Statement of the problem}

As an astronomical instrument, SVLBI possesses one explicit advantage: it has an extremely high angular resolution, which currently could be potentially up to $10^{-6}$ arcsec, but it has a potentially unlimited prospect. The disadvantages for SVLBI observations are:

1. phase instability;

2. poor $(u, v)$-coverage;

3 . low sensitivity.

Possible ways to solve these problems could be:

1. use of Multi-Frequency Synthesis (MFS) that will enable us to improve poor $(u, v)$-coverage, dynamic range, and phase instability;

2. use of sensitive radio telescopes or compact arrays as ground support for SVLBI missions that will enable us to improve the sensitivity of the SVLBI mission;

3. use of two or more space-borne radio telescopes to improve poor $(\mathrm{u}, \mathrm{v})$ coverage and sensitivity;

4. developing a modern imaging technology.

It is possible to show that for a High Orbiting SVLBI mission any two spaceground baselines are almost equal to each other i.e., $\left\|\mathbf{b}_{1}\right\| \approx\left\|\mathbf{b}_{2}\right\|=\|\mathbf{b}\|$ and consequently for a ground-based baseline (consisting of two ground-based radiotelescopes (GRT)) a phase difference can be small enough, i.e., $\Delta \varphi_{G R T 1, G R T 2} \rightarrow 0$. Generally speaking, $\Delta \varphi_{G R T 1, G R T 2}$ can be comparable with a phase error (i.e., $\Delta \varphi_{G R T 1, G R T 2} \approx \delta \varphi_{r m s}$ ) that can be a reason of phase instability and possible phase breakup in apogee part of the high orbit. It is possible to make another 
useful outcome: in the case of a High Orbiting SVLBI mission, a good $(\mathrm{u}, \mathrm{v})$ coverage doesn't guarantee high quality images.

Since a high Space Radio Telescope (SRT) orbit can be evaluated in realtime, it is possible to use the part of the orbit with the well-estimated SRT parameters to provide a better estimate of the SRT parameters in other parts of the orbit. There are three main items of space mission data adaptation that should be considered:

1. Pre-launch surveys - define a set of the targets for future investigations (a set of radio sources), and possible source structures that can be specified by the space mission.

2. Short-term orbiting adaptation method (OAM) uses perigee information about a radio source as a prior for estimating the source parameter for other parts of the orbit (i.e. initial model for the Hybrid mapping can be taken from "good" parts of an orbit and used for apogee observations of this source);

3. Long-term OAM - uses long-term evaluation of the SRT orbit (weeks, months, years) to improve $(u, v)$-coverage for observations of the same source.

\section{Basic outcomes}

1. Current VLBI technology enables us to obtain high quality images for SVLBI missions having an apogee comparable with Moon orbit.

2. Since for SVLBI missions with very high orbits the multi-baseline interferometer transfers to a one baseline interferometer, it is not necessary to provide a powerful set of ground based radio telescopes to support a new high Radioastron orbit.

3. It is necessary to provide powerful and reliable tracking stations, digital registration and correlation of the Radioastron data to avoid (or to minimize) possible phase errors.

4. From the imaging point of view, the procedure of Fringe Fitting and Self Calibration (Hybrid mapping) must be revised to take into account the data obtained from the apogee part of the Radioastron high orbit.

5. The Orbiting Adaptation Method (OAM) allows us essentially to improve both procedures.

6. For high orbiting SVLBI, deconvolution methods of extended sources have to take into account the relationship $\frac{\text { SourceSize }}{\text { BeamWidth }}$.

7. The future of VLBI lies in the high orbiting (interplanetary) Space VLBI missions having a few SRTs. 